\title{
On the role of substrate in light-harvesting experiments
}

\author{
Ilya Sychugov,* Hiroo Omi, and Yoshihiro Kobayashi \\ NTT Basic Research Laboratories, NTT Corporation, Atsugi, Kanagawa 243-0198, Japan \\ *Corresponding author: ilya@will.brl.ntt.co.jp
}

Received May 28, 2008; revised July 7, 2008; accepted July 7, 2008;

posted July 14, 2008 (Doc. ID 96306); published August 6, 2008

\begin{abstract}
An analysis of the emitted light distribution for a single emitter located at the planar interface of two optical media was performed. The interface of a varying refractive index substrate with air was considered, which is a common case in luminescence microscopy (spectroscopy) experiments. A modification of the radiative recombination rate induced by the variation of the substrate together with the emitted radiation spatial redistribution were taken into account. Simulation results show that the collection efficiency of the emitted light can vary several times depending on the substrate choice and the emitter intrinsic quantum efficiency. (C) 2008 Optical Society of America

OCIS codes: $250.5230,260.3800$
\end{abstract}

An often used configuration for a luminescence microscopy (spectroscopy) experiment is when an investigated object is located at the interface between a substrate and air. The probed particles, such as nanocrystals [1] or nanotubes [2], are excited with an external source, and their luminescence is collected in the upper or lower (providing the substrate is transparent) half-space. Such a time-integrated method is employed in, for example, photoluminescence spectroscopy, microphotoluminescence, near-field optical microscopy, etc. The spatial distribution of the emitted light in this configuration depends on the substrate optical properties. An understanding of the substrate's role can help in improving the collection of light from a weakly emitting source. In addition, a deviation from an isotropic spatial distribution of the emitted light should be taken into account for the emitter quantum-efficiency estimation.

Previously the substrate effect was experimentally observed for a subwavelength ensemble of Si nanocrystals located on pillars etched various distances from the semiconductor substrate [3]. It was shown that the luminescence yield can be enhanced and the radiative lifetime can be increased by reducing the density of optical modes below the emitter. Here we discuss a general case for a single emitter at the interface and calculate its emission spatial distribution. Numerical simulations were performed using the finite-element method (FEM) for radiative power distribution, and the change in the corresponding radiative rate was taken into account using its proportionality to the density of optical modes at the emitter location. We consider a spontaneous radiative transition for a single emitter from the excited $\left(X^{*}\right)$ to the ground $(X)$ state with a photon emission [4]:

$$
X^{*} \rightarrow X+h \nu .
$$

The final state of this transition consists of the emitter in its ground state and the propagating electromagnetic mode; the latter is necessary for energy conservation. In general, according to Fermi's golden rule a transition rate is proportional to the density of available final states [5]. Thus the radiative transi- tion rate can be changed by modifying the number of photonic modes, supported by the local optical environment. This fact was first pointed out by Purcell [6], and the modification of the spontaneous emission rate induced by the radiative properties of the environment is often referred to as the Purcell effect. Thus the radiative transition rate is proportional to the local density of optical modes (LDOS), which is, in turn, linearly dependent on the medium refractive index at the emitter location [7]. In addition, a reflected wave from the interface affects LDOS and, consequently, the radiative rate for an emitter at a distance from the interface [8]. When an emitter is considered at the interface of two optical media, however, the LDOS calculations encounter a strong singularity with diverging solutions [7]. Here we assume that the emitter radiative rate $\Gamma_{\mathrm{rad}}$ is proportional to the mean value of corresponding refractive indices (Fig. 1). In such a representation a radiative rate for a pointlike emitter located at the interface between air $\left(n_{\text {air }}=1\right)$ and a substrate (refractive index $n$ ) can be expressed as

$$
\Gamma_{\mathrm{rad}}=\frac{n+1}{2} \Gamma_{0 \mathrm{rad}}
$$

where $\Gamma_{0 \text { rad }}$ is a radiative transition rate for the emitter suspended in vacuum, i.e., the intrinsic radiative rate. Thus when the substrate is a semiconductor [Fig. 1(a)] the radiative rate is stronger than in the case of silica substrate [Fig. 1(b)]. On the other hand, a nonradiative transition rate is nearly independent of a change in LDOS. The rate $\Gamma_{n r}$ for the nonradiative transition,

$$
X^{*} \rightarrow X+\text { heat, }
$$

is rather defined by the emitter internal characteristics: $\Gamma_{n r}=\Gamma_{0 n r}$. Then the luminescence quantum efficiency $\mathrm{QE}$, i.e., the fraction of total power going to the radiative channel, for the emitter at the airsubstrate interface is 


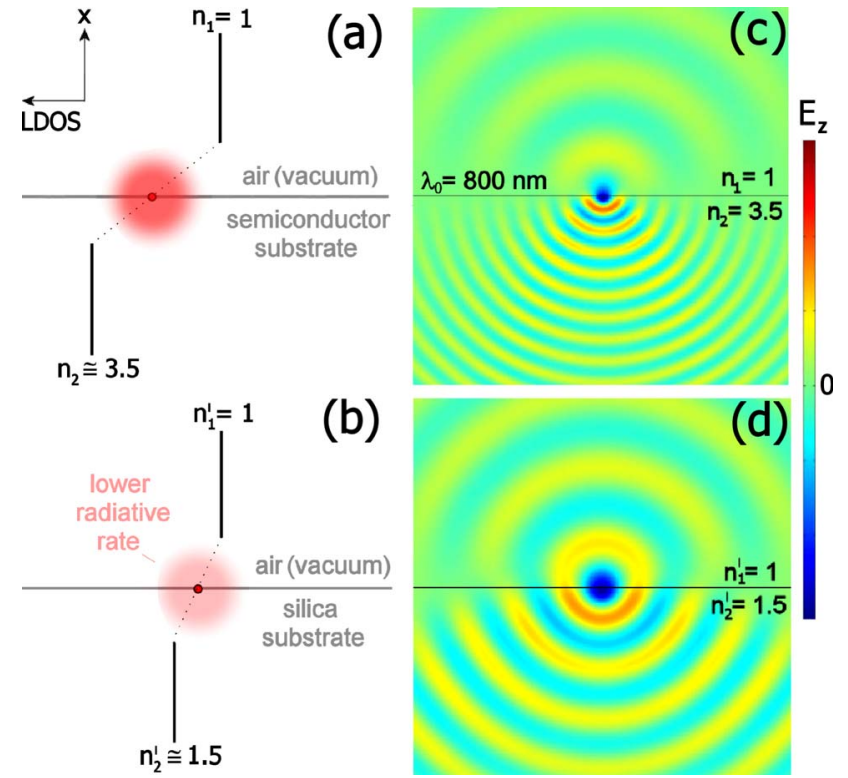

Fig. 1. (Color online) (a), (b) Schematic representation of the interface effect on the emitter radiative rate. (a) For a pointlike emitter located at the air-semiconductor interface the radiative rate is higher than (b) for the one located at the air-silica interface. (c), (d) Electric field $z$-component distribution for TE-polarized light with free-space wavelength $\lambda_{0}=800 \mathrm{~nm}$, emitted from a single emitter located at the (c) air-semiconductor and (d) air-silica interfaces.

$$
\mathrm{QE}=\frac{\Gamma_{\mathrm{rad}}}{\Gamma_{\mathrm{rad}}+\Gamma_{n r}} \equiv \frac{n+1}{n-1+2 / \mathrm{QE}_{0}},
$$

where the emitter intrinsic quantum efficiency $\mathrm{QE}_{0}$ $=\Gamma_{0 \mathrm{rad}} /\left(\Gamma_{0 \mathrm{rad}}+\Gamma_{0 n r}\right)$.

The spatial distribution of this radiative power, in turn, also depends on a particular optical environment. For geometry with rotational symmetry a three-dimensional (3D) task can be considered as a two-dimensional (2D) case with a fixed field polarization. Then the propagating wave equation for a sinusoidal monochromatic source can be reduced to the Helmholtz equation [9]

$$
\Delta E+k_{0}^{2} n^{2} E=0
$$

where $k_{0}=2 \pi / \lambda_{0}, \lambda_{0}$ is the emission wavelength in vacuum and $E=E_{z}$ is the electric field transverse component for the TE polarization of the emitted light (i.e., the dipole is parallel to the substrate plane). Numerical solutions to this equation for a subwavelength source located either on a semiconductor substrate or on a silica substrate are shown in Figs. 1(c) and 1(d). This field distribution was obtained using the FEM, embedded into the FEMLAB package. It was shown that the FEM can yield results for a given wavelength significantly faster than the finite-difference time-domain (FDTD) calculation method [10]. The source was defined as having a fixed amplitude and, for certainty, emitting at $\lambda_{0}$ $=800 \mathrm{~nm}$. It is seen that in the case of a silica substrate [Fig. 1(d)] the electric field amplitude in the upper half-space is larger than in the case of a semiconductor substrate [Fig. 1(c)]. Then, the light inten- sity emitted in the upper or lower half-space can be calculated using the obtained electric field distribution. It was done by integrating the Poynting vector over the corresponding boundary. Thus a fraction of the radiative power emitted to the upper $\chi_{\text {rad }}^{\uparrow}$ and lower $\chi_{\text {rad }}^{\uparrow}$ half-space can be estimated.

Then the fraction of total power distributed to the upper $\kappa^{\uparrow}$ or lower $\kappa^{\downarrow}$ half-space is a product of the quantum efficiency (4) and these coefficients $\chi_{\mathrm{rad}}^{\uparrow}$, $\chi_{\text {rad }}^{\downarrow}$ :

$$
\kappa^{\uparrow, \downarrow}\left(n, \mathrm{QE}_{0}\right)=\mathrm{QE}\left(n, \mathrm{QE}_{0}\right) \chi_{\mathrm{rad}}^{\uparrow, \downarrow}(n)
$$

The results of these calculations are presented in Figs. 2 and 3. In Fig. 2(a) the coefficient $\kappa^{\uparrow}$ is shown as a function of the lower half-space refractive index $n$ for different values of the emitter intrinsic quantum efficiency $\mathrm{QE}_{0}$. In Fig. 3(a) this coefficient is depicted depending on the emitter intrinsic quantum efficiency for three different values of the lower halfspace refractive index $n=1,1.5,3.5$. The latter two values represent practically important cases of fused silica (quartz) and semiconductor substrates. The coefficient $\kappa^{\downarrow}$ is shown in Figs. 2(b) and 3(b) in the same manner.

The fraction of power emitted to the upper or lower half-space $\kappa^{\uparrow, \downarrow}$ changes steeply when deviating from

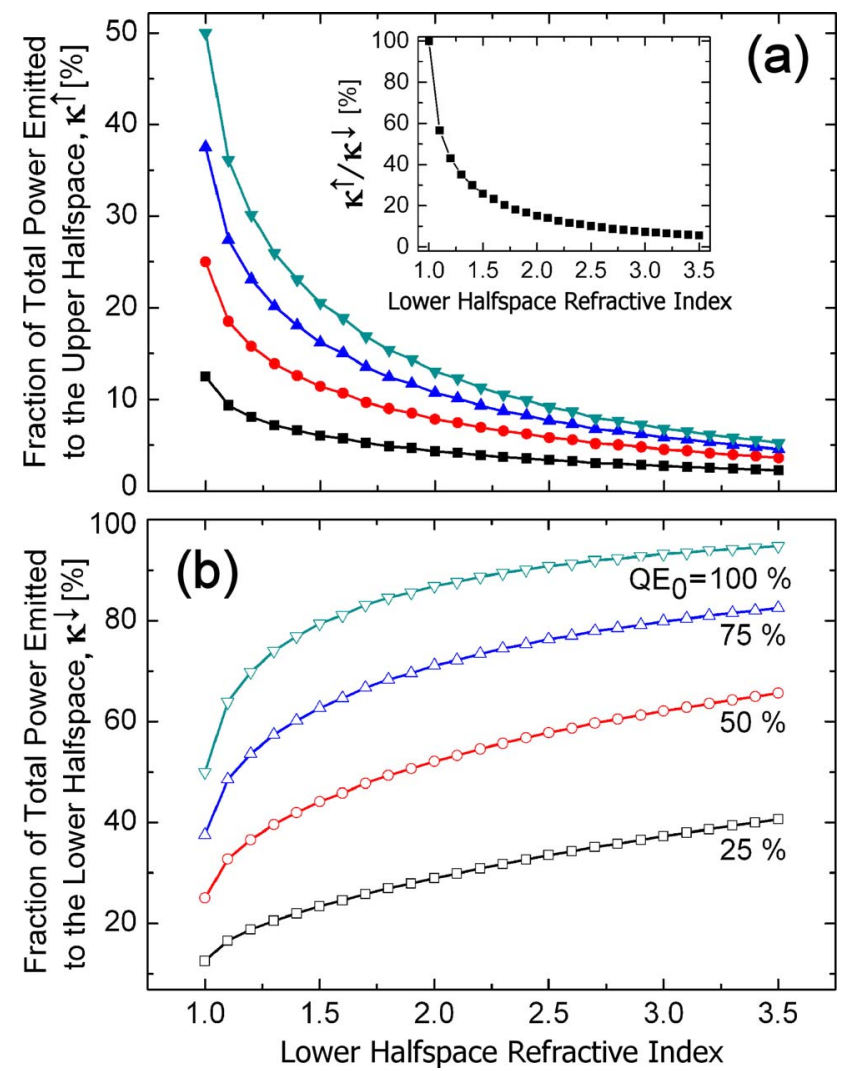

Fig. 2. (Color online) Fraction of total power emitted to the (a) upper and (b) lower half-space as a function of lower half-space refractive index for several values of the emitter intrinsic quantum efficiency $\mathrm{QE}_{0}: 25 \%, 50 \%, 75 \%$, and $100 \%$. Inset, the ratio of total power emitted to the upper and lower half-space as a function of substrate refractive index. 


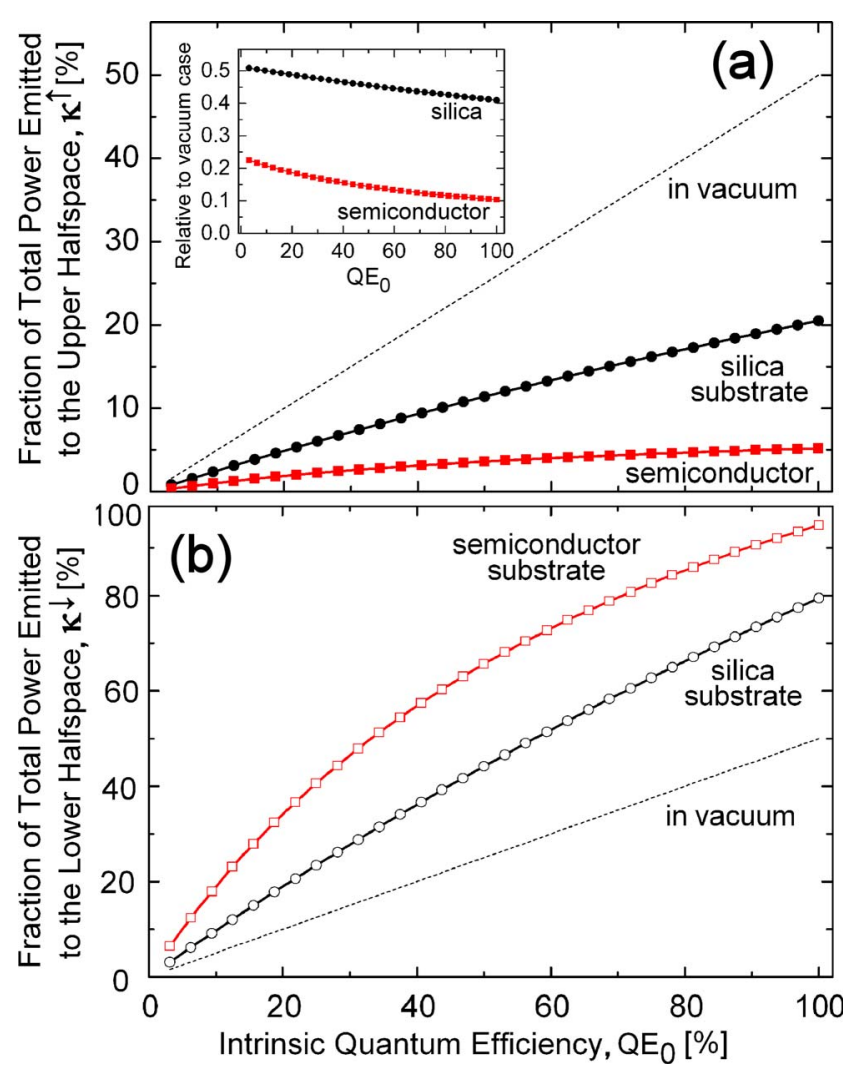

Fig. 3. (Color online) Fraction of total power emitted to the (a) upper and (b) lower half-space as a function of the emitter intrinsic quantum efficiency for the common case of silica and semiconductor substrates. The case of isotropic distribution for an emitter suspended in vacuum is shown with a dotted line. Inset, curves for the upper half-space are shown relative to the case of isotropic emission.

the refractive index $n=1$ as seen in Fig. 2, inset. The value of $\kappa^{\uparrow}$, for example, decreases two times when the substrate refractive index increases to the value $n=1.4$ [Fig. 2(a)]. Practically it implies that any substrate does remarkably change the spatial distribution of the total power with the refractive indices starting from 1.3-1.4 for most optical materials [11].

The substrate effect becomes less pronounced at smaller emitter intrinsic quantum efficiencies for the light collection in the upper half-space [Fig. 3(a)]. For example, the fraction of the total power emitted to the upper half-space increases in relative units for the case of the semiconductor substrate from 0.1 for $\mathrm{QE}_{0}=100 \%$ to 0.2 for $\mathrm{QE}_{0}=10 \%$ (Fig. 3, inset). This is due to the nonradiative recombination, which effectively screens the effect of spatial redistribution of the radiative power induced by the substrate modification. In practice, placing an emitter on a fused silica substrate can improve the signal, collected in the upper half-space, up to 4 times compared to a semiconductor substrate (Fig. 3 , inset). This value changes to 2.5 for an emitter with $\mathrm{QE}_{0}=10 \%$ owing to the intervention of the nonradiative recombination process. Experimentally, an increase in the luminescence yield by a factor of 2.5 was detected for Si nanocrystals distanced from the silicon substrate [3]. When the nanocrystals are etched from the semiconductor substrate the refractive index of the lower half-space can be considered as being effectively close to the case of a silica substrate.

As a digression from the present calculations one can note that the same effect takes place in the nearfield optical microscopy. In a similar way as the substrate affects emitter performance a near-field optical probe capable of either electrical or optical excitation [12] modifies the emitter optical environment. Although the tip is not necessarily in physical contact with the emitter, the density of optical modes around the emitter becomes altered with the tip introduction.

In brief, we carried out numerical simulations for a general case of an emitter located at the airsubstrate interface with the focus on power spatial distribution. Redistribution of the radiative power caused by the substrate modification as well as the radiative transition rate change were taken into account. The results indicate that the luminescence yield can vary several times depending on the substrate and emitter intrinsic quantum efficiency. Thus the effect of the substrate should not be ignored for this class of experiments with regard to the collection efficiency enhancement or intrinsic quantum efficiency estimations.

We thank Jan Linnros of the Royal Institute of Technology in Stockholm for a stimulating discussion and Koji Muraki of NTT Basic Research Labs for access to the FEMLAB simulation package.

\section{References}

1. S. A. Empedocles, D. J. Norris, and M. G. Bawendi, Phys. Rev. Lett. 77, 3873 (1996).

2. H. Htoon, M. J. O'Connel, P. J. Cox, S. K. Doorn, and V. I. Klimov, Phys. Rev. Lett. 93, 027401 (2004).

3. I. Sychugov, A. Galeckas, N. Elfström, A. R. Wilkinson, R. G. Elliman, and J. Linnros, Appl. Phys. Lett. 89, 111124 (2006).

4. N. J. Turro, Modern Molecular Photochemistry (Benjamin/Cummings, 1978), p. 298.

5. L. A. Coldren and S. W. Corzine, Diode Lasers and Photonic Integrated Circuits (Wiley, 1995), p. 509.

6. E. M. Purcell, Phys. Rev. 69, 681 (1946).

7. E. Snoeks, A. Lagendijk, and A. Polman, Phys. Rev. Lett. 74, 2459 (1995).

8. R. Amos and W. L. Barnes, Phys. Rev. B 55, 7249 (1997).

9. D. W. Dearholt and W. R. McSpadden, Electromagnetic Wave Propagation (McGraw-Hill, 1973), p. 402.

10. T. Grosges, A. Vial, and D. Barchiesi, Opt. Express 13, 8483 (2005).

11. H. L. Anderson, A Physicist's Desk Reference (American Institute of Physics, 1981), p. 272.

12. I. Sychugov, H. Omi, T. Murashita, and Y. Kobayashi, Appl. Surf. Sci. (to be published). 